\title{
Monitoring the Diffusion Layer During Passive Film Breakdown on Alloy 800 with Digital Holography
}

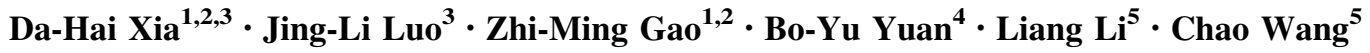

Received: 4 May 2015/Revised: 29 June 2015/Published online: 8 September 2015

(C) The Chinese Society for Metals and Springer-Verlag Berlin Heidelberg 2015

\begin{abstract}
The effects of chloride and thiosulfate ions on localized corrosion of alloy 800 are investigated through dynamical observation of the change in phase image of the diffusion layer during passive film breakdown using digital holography. The results indicate that solution chemistry has a significant effect on film breakdown and diffusion layer. The phase distribution changes at different applied potentials show that in the process of film breakdown, dissolution of metal ions from pitting is not remarkable in chloride-only solution, whereas dissolution of metal ions is significantly high in thiosulfate and chloride solution. Thiosulfate has a combined effect with chloride ions in passive film degradation.
\end{abstract}

KEY WORDS: Passive film; Breakdown; Diffusion layer; Digital holography; Alloy 800

\section{Introduction}

Passive film breakdown is a very important electrochemical process which occurs on the metal and alloy surface, because it is usually the initial stage of pitting corrosion and stress corrosion cracking (SCC) for self-passive pure

Available online at http://link.springer.com/journal/40195

Da-Hai Xia

dahaixia@tju.edu.cn

$\triangle$ Jing-Li Luo

luoj@ualberta.ca

1 Tianjin Key Laboratory of Composite and Functional Materials, Tianjin University, Tianjin 300072, China

2 School of Materials Science and Engineering, Tianjin University, Tianjin 300072, China

3 Department of Chemical and Materials Engineering, University of Alberta, Edmonton, AB T6G 2V4, Canada

4 School of Physics and Electronic Engineering, Jiangsu Normal University, Xuzhou 221116, China

5 School of Chemistry and Chemical Engineering, Jiangsu Normal University, Xuzhou 221116, China metals and alloys $[1,2]$. Because passive film breakdown is an electrochemical process at nanoscale or micro-nanoscale and is usually very localized, traditional electrochemical methods, including polarization curve [3, 4], electrochemical impedance spectroscopy (EIS) [5-7] and electrochemical noise (EN) [8], are not able to capture some localized activities of this process. Therefore, researchers have applied localized electrochemical methods to explore this process. For example, Cui et al. [9] used scanning reference electrode to measure the surface potential distribution. Zhu et al. [10, 11] and Xia et al. [12, 13] used scanning electrochemical microscope (SECM) to obtain the surface reactivity of alloy 800 in chloride and thiosulfate solutions. Zhao et al. [14] used Scanning Kelvin Probe (SKP) to study the potential distribution of a crack on 304 stainless steel. However, passive film breakdown is a complex process including dissolution of metal and diffusion of reactant and corrosion product. Thus, dynamically tracking or monitoring the activities in diffusion layer during passive film breakdown can provide new insights into this process and may help us understand the detailed mechanism of the breakdown process.

Digital holography is a new optical method which offers an easy, useful, nondestructive way to in situ observe the 
dynamic changes on an electrode/electrolyte interface. $\mathrm{Li}$ et al. [15] investigated current oscillations during pitting corrosion of Fe using digital holography. Yuan et al. [16] observed the anodic dissolution of copper in $\mathrm{NaCl}$ solution with the digital holography and proposed anodic dissolution mechanism. Though the anodic dissolution of metal and alloys has been studied using digital holography, the technique has not been used to investigate the passive film breakdown. Therefore, the aim of this work is to investigate the passive film breakdown process using digital holography.

\section{Experimental}

The specimens were prepared using alloy 800 SG tubing (Sandvik, heat number 516809, outer diameter of $15.88 \mathrm{~mm}$, wall thickness of $1.13 \mathrm{~mm}$ ) [17]. The composition of the alloy 800 is listed in Table 1 . The tube was cut into a long strip, the front-end was ground to very flat surfaces, and the exposed area was about $0.2 \mathrm{~cm}^{2}$. Cyclic polarization was conducted at $21{ }^{\circ} \mathrm{C}$ at $10 \mathrm{mV} / \mathrm{s}$ by using a CHI660B electrochemical station. A three-electrode cell was used with the alloy 800 as the working electrode, a saturated calomel electrode (SCE) as the reference electrode and a platinum electrode as the counter electrode.

For the holographic recording system, a well-established digital camera (Sony DSRPD150P) was used to record the dynamical process. Holograms that correspond to various states of the alloy $800 /$ solution interface were processed by numerical methods for measurement. The dynamic processes of electrochemical reactions can be studied from the consecutive interferograms which were stored as AVI (audio visual interleaved) files at up to 25 frames per second in a personal computer. In processing, software was developed with MATLAB R2007b for the reconstruction of the phase maps from the AVI video signals [18]. The initial interferogram at the time when the reaction began was selected, and the distribution of phase difference was continually displayed on the PC monitor. During the holography experiment, the change in the phase of the object beam is determined by the change in the refractive index inside the cell, which is associated with the changes in concentration caused by the electrochemical reaction. The principle of the experiment is based on the relationships between the phase difference $(\Delta \phi)$, the solution refractive index $(\Delta n)$ and the solution concentration $(\Delta C)$, which was expressed as [18]:

$\Delta C=k \Delta n=\frac{k \lambda_{0} \Delta \phi}{2 \pi d}$.

where $k$ is the concentrative refractivity, $\lambda_{0}$ is the wavelength of the laser light, $d$ stands for the geometrical path length where the refractive index variation exists. Generally, several components are present in the solution. Thus, the net refractive index is the summation of the effect of each species' concentration:

$\sum_{i}\left(\Delta C_{i}\right)=\sum_{i}\left(\Delta k_{i} \Delta n_{i}\right)=\frac{\lambda_{0}}{2 \pi d} \sum_{i}\left(k_{i} \Delta \phi_{i}\right)$.

\section{Results and Discussion}

The cyclic polarization curve of alloy 800 in $0.6 \mathrm{~mol} / \mathrm{L}$ $\mathrm{NaCl}$ solution is shown in Fig. 1. Self-passive behavior of alloy 800 was monitored in $\mathrm{NaCl}$ solution, and the passive film was stable and was not broken down when the potential was lower than $0.4 \mathrm{~V}_{\mathrm{SCE}}$. However, above 0.4 $\mathrm{V}_{\mathrm{SCE}}$, the current increased due to film breakdown and many transient peaks could be found in the curve, which is attributed to the metastable pit initiation and repassivation [19]. Previous results indicated that passive film formed on alloy 800 in chloride-only solution is not easy to broken down unless the anodic polarization potential value is high enough, and the pitting propagation rate is also very low because the pits size in this solution is very tiny and sometimes even cannot be identified [17, 19].

A selection of quantitative phase images, reconstructed at various potentials which are marked in the cyclic polarization curve (Fig. 1), is shown in Fig. 2, which depicts the distribution of phase differences at various potentials. As shown in the distribution of phase difference, the left sides are the alloy 800 electrode, whereas the right sides are the electrolyte. The interfaces can be identified by the boundary between the electrode and electrolyte. It is evident that the color variation appears only at the interface where color changing to yellow or red means the increase in the phase difference, while changing to blue means the decrease [20]. The green area in Fig. 2 indicates that the phase difference was zero or almost zero. When the passive film was intact and undamaged under certain potentials, such as point $\mathrm{A}$, the phase color was green, which means there was no detected

Table 1 Chemical compositions of alloy 800 (in wt $\%$ )

\begin{tabular}{llllllllllllll}
\hline $\mathrm{C}$ & $\mathrm{Si}$ & $\mathrm{Mn}$ & $\mathrm{P}$ & $\mathrm{S}$ & $\mathrm{Cr}$ & $\mathrm{Ni}$ & $\mathrm{Co}$ & $\mathrm{Ti}$ & $\mathrm{Cu}$ & $\mathrm{Al}$ & $\mathrm{N}$ & $\mathrm{Fe}$ \\
\hline 0.017 & 0.46 & 0.5 & 0.012 & 0.001 & 21.87 & 32.78 & 0.01 & 0.48 & 0.02 & 0.29 & 0.016 & 43.2 \\
\hline
\end{tabular}




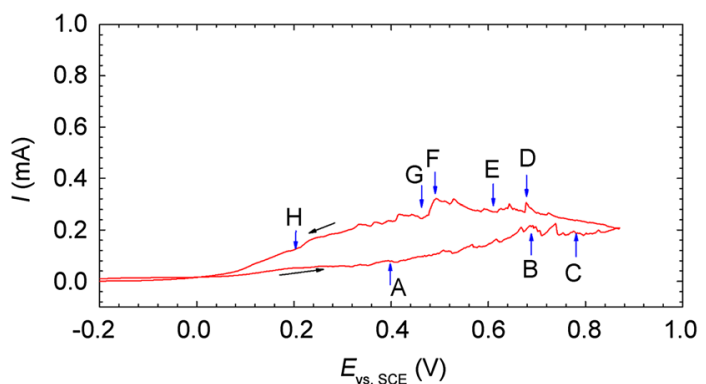

Fig. 1 Cyclic voltammogram of the alloy 800 in $0.6 \mathrm{~mol} / \mathrm{L} \mathrm{NaCl}$ solution with the scan rate of $10 \mathrm{mV} / \mathrm{s}$

phase difference in this stage, as shown in Fig. 2a. As the potential increased to points $\mathrm{B}$ and $\mathrm{C}$, a region with yellow color could be seen in the phase distribution (Fig. 2b and c); therefore, the corresponding region on the alloy 800 surface was exactly the site where passive film was broken down, as a result of $\mathrm{Fe}^{2+}, \mathrm{Cr}^{3+}$ and $\mathrm{Ni}^{2+}$ generated from the dissolution reactions (3) to (5). The release of $\mathrm{Fe}^{2+}, \mathrm{Cr}^{3+}$ and $\mathrm{Ni}^{2+}$ increased the concentrations of these ions at the interface and resulted in an increase in the refractive index. Subsequently, the phase difference became positive. For points D, E, F and G in Fig. 1, the dissolution rate increased due to an increase in anodic current; therefore, the refractive index continued to increase, as shown in Fig. 2d-g. As the potential decreased to the point $\mathrm{H}$ (about $0.2 \mathrm{~V}_{\mathrm{SCE}}$ ), pits were repassivated and the generated cations diffused into the bulk solution, so the phase difference decreased accordingly, as shown in Fig. $2 \mathrm{~h}$.

$\mathrm{Fe} \rightarrow \mathrm{Fe}^{2+}+2 \mathrm{e}^{-}$.

$\mathrm{Cr} \rightarrow \mathrm{Cr}^{3+}+3 \mathrm{e}^{-}$.

$\mathrm{Ni} \rightarrow \mathrm{Ni}^{2+}+2 \mathrm{e}^{-}$.

The cyclic polarization curve of alloy 800 in $0.6 \mathrm{~mol} / \mathrm{L}$ $\mathrm{NaCl}+0.075 \mathrm{~mol} / \mathrm{L} \mathrm{Na} \mathrm{S}_{2} \mathrm{O}_{3}$ solution is shown in Fig. 3,

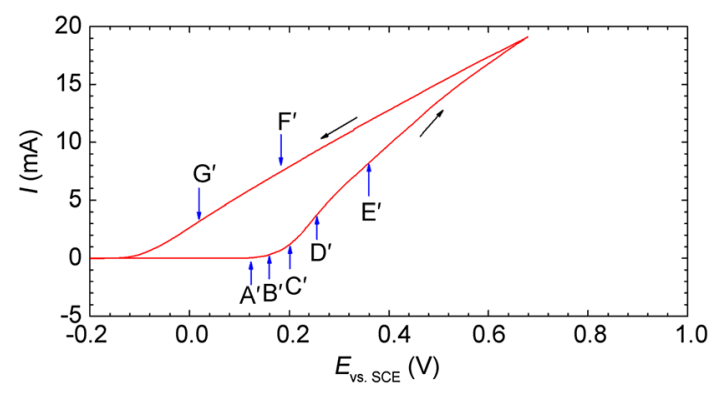

Fig. 3 Cyclic voltammogram of the alloy 800 in $0.6 \mathrm{~mol} / \mathrm{L}$ $\mathrm{NaCl}+0.075 \mathrm{~mol} / \mathrm{L} \quad \mathrm{Na}_{2} \mathrm{~S}_{2} \mathrm{O}_{3}$ solution with the scan rate of $10 \mathrm{mV} / \mathrm{s}$

and the corresponding phase distributions at various potentials are shown in Fig. 4. Similar to the analyses for Fig. 2a, for point $\mathrm{A}^{\prime}$ at about $0.12 \mathrm{~V}_{\mathrm{SCE}}$, the phase color of the electrolyte was green, indicating that there was no detected phase difference in this stage, and the passive film was intact under $0.12 \mathrm{~V}_{\mathrm{SCE}}$ (Fig. 4a). As the potential increased to $0.15 \mathrm{~V}_{\mathrm{SCE}}$ (point $\mathrm{B}^{\prime}$ ), the current began to increase, resulting in breakdown of the passive film (Fig. 4b). Obviously, the pitting potential in solution containing thiosulfate was about $200 \mathrm{mV}$ lower than that in thiosulfate-free solution. As it was explained previously, the reason is that thiosulfate reduces the pitting potential by adsorption within the metastable pits and stabilizes the metastable pitting induced by chloride ions [21]; therefore, thiosulfate could block repassivation in chloride solutions. Newman et al. [22] found the same phenomenon for 304 $\mathrm{SS}$ in $0.25 \mathrm{~mol} / \mathrm{L} \mathrm{NaCl}$ solution containing various concentrations of thiosulfate. They claimed that the combined effect of these two ions is due to the breakdown of passive film by chloride ions and accumulation of thiosulfate at rapidly dissolving pit
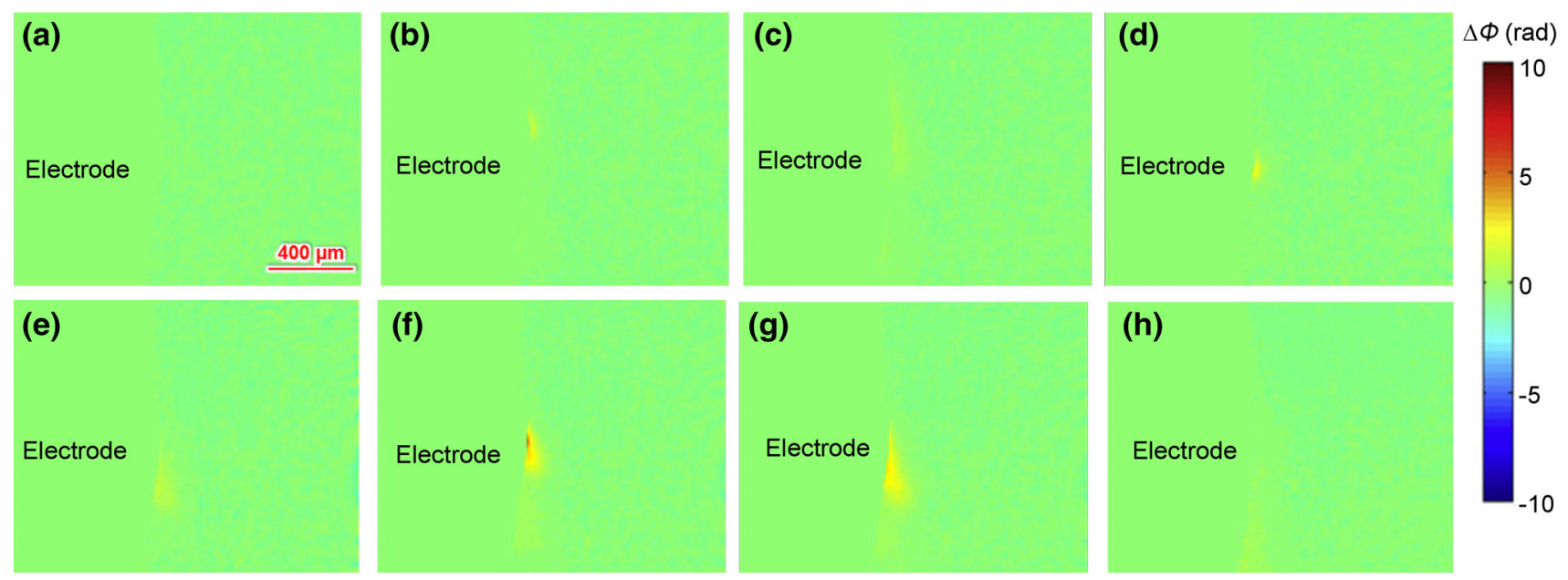

Fig. 2 Corresponding phase maps at various polarization potentials that marked by letters A a, B b, C c, D d, E e, F f, G g and H h in Fig. 1 


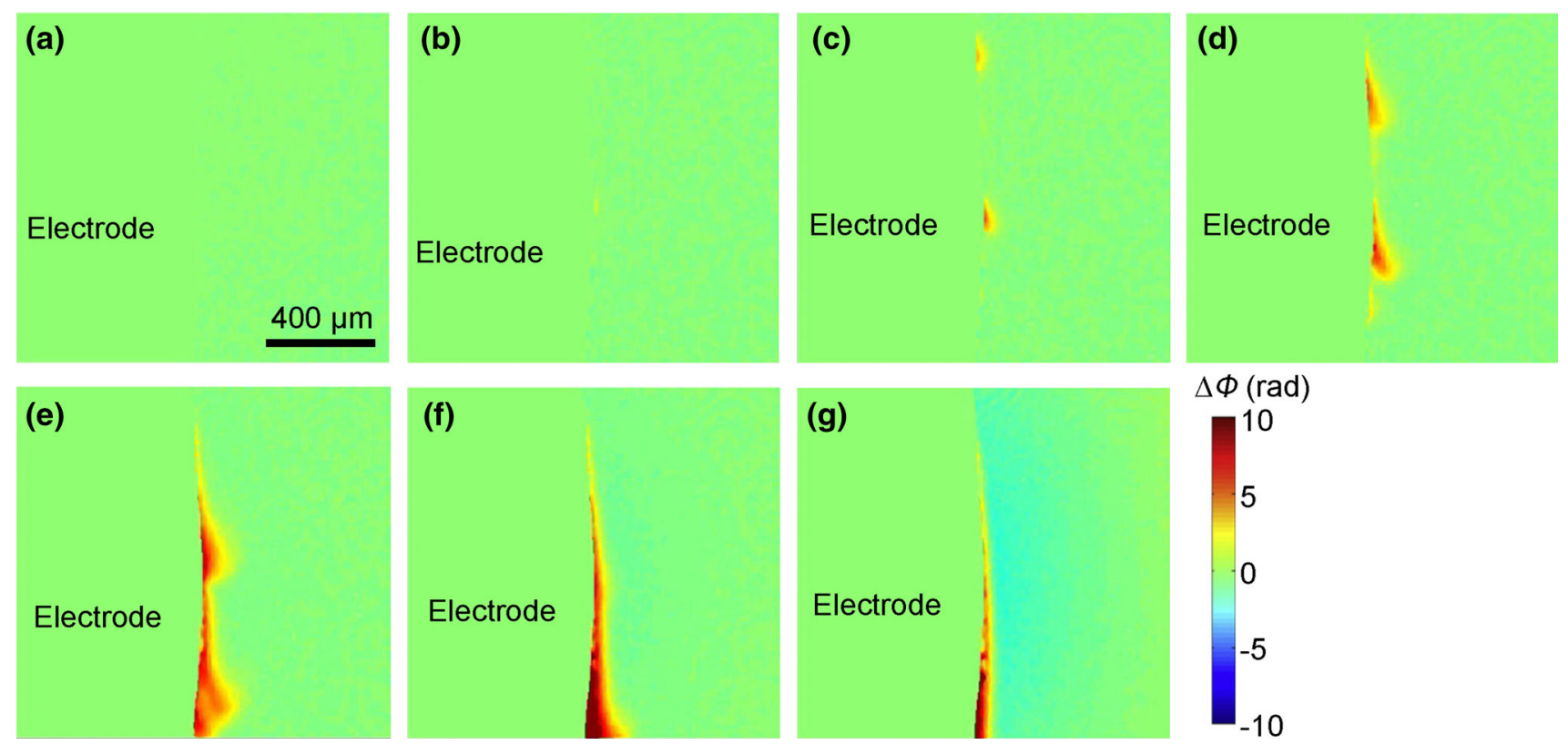

Fig. 4 Corresponding phase maps at various polarization potentials that marked by letters $A^{\prime} \mathbf{a}, B^{\prime} \mathbf{b}, C^{\prime}$ c, $D^{\prime}$ d, $E^{\prime}$ e, $F^{\prime}$ f, G $G^{\prime}$ g in Fig. 3

precursors to form $\mathrm{S}^{0}$ which would stabilize metastable pits and accelerate anodic dissolution. As the potential continued to increase, the dissolution rate also increased at the pitting sites, resulted in that the concentration of these ions close to the interface became high, so the phase color became red, as shown in Fig. 4c-f.

Comparing Figs. 1 and 2 with Figs. 3 and 4, it is concluded that the dissolution of metal ions is not remarkable in thiosulfate-free solution, whereas the dissolution of metal ions is significant in thiosulfate-containing solution, which is due to thiosulfate-enhanced anodic dissolution within the pits, and this is consistent with the previous experimental observations [19, 23]. Moreover, the current in thiosulfate-containing solution is significantly higher than that in thiosulfate-free solution. Therefore, the thiosulfate played an important role in the passive film breakdown by adsorption on the metastable pits to catalyze anodic dissolution. Thiosulfate has a sulfur chain that is uncapped. The uncapped ends are free radicals containing lone, unpaired electrons, which render them very reactive, especially toward bare metal surfaces, such as copper, nickel and iron, provided that the configuration of the adsorbed species allows access of the uncapped end to the metal atoms [20]. In addition, the pitting propagation rate was much higher in thiosulfate-containing solution than that in thiosulfate-free solution, which was attributed to the catalytic anodic dissolution caused by thiosulfate.

Figure 5 shows the surface morphologies of the samples after the cyclic polarization test. The pit size in thiosulfatefree solution is small and in the range of several micrometers, but the pit size in thiosulfate-containing solution was about $100 \mu \mathrm{m}$, indicating that the pitting propagation rate in thiosulfate and chloride solution was significantly higher than that in thiosulfate-free solution. Apart from the large pitting damage shown in Fig. 5b, there are also many tiny pits over the sample surfaces (Fig. 5a, b), but these pits were not recorded in the holographic photographs because the dissolution of metal ions within these small pits is very limited, so they were beyond the detecting limit.

In fact, the effects of the thiosulfate and chloride ions on film degradation depended on the concentration ratio of these two ions, and the combined effect can be observed at high concentration ratios of chloride to thiosulfate; at low concentration ratios, the adsorption of thiosulfate is dominant so that there is no opportunity for chloride ions to adsorb onto the surface to damage the passive film. Therefore, no pits are initiated at a lower chloride-tothiosulfate concentration ratio $[12,21]$. The concentration of these two ions could affect the semiconductivity of passive film formed on alloy 800 , and the semiconductivity type changes from n-type to p-type as the concentration ratio decreased, which was due to sulfur incorporation into oxygen vacancies [21].

In summary, the solution chemistry plays an important role on film breakdown, pitting propagation rate and diffusion layer. Digital holography is a new method that helps us to better understand this process and the related mechanism. Through observing the diffusion layer changes during film breakdown, it was found that dissolution of metal ions at the pits is not remarkable in chloride-only solution, whereas dissolution of metal ions is significant in thiosulfate and chloride solution. 

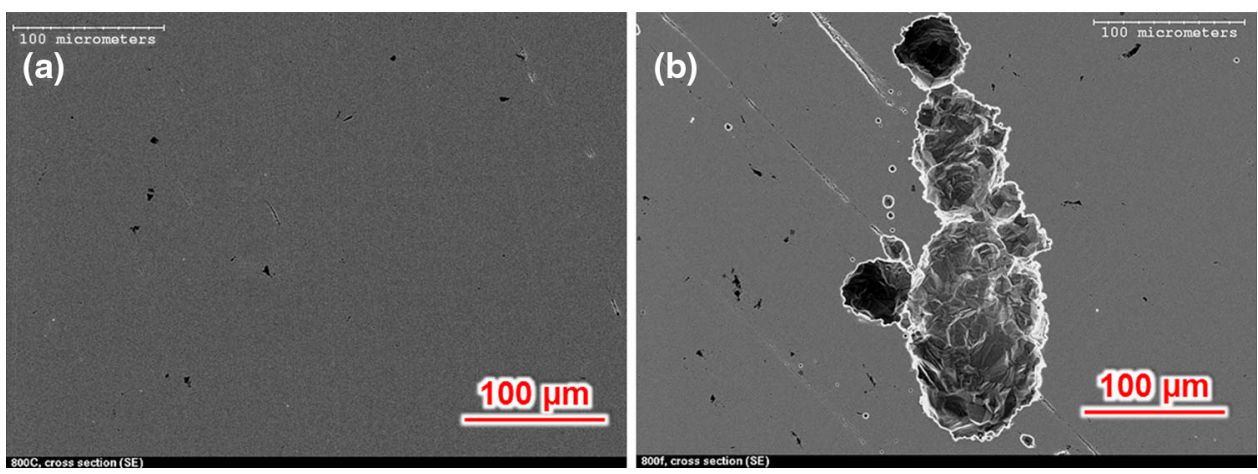

Fig. 5 SEM images of alloy 800 after testing in $0.6 \mathrm{~mol} / \mathrm{L} \mathrm{NaCl}$ solution a and $0.6 \mathrm{~mol} / \mathrm{L} \mathrm{NaCl}+0.075 \mathrm{~mol} / \mathrm{L} \mathrm{Na} \mathrm{S}_{2} \mathrm{O}_{3}$ solution b

\section{Conclusions}

The change in phase image of the diffusion layer during passive film breakdown was dynamically observed using digital holography. The results showed that the addition of a small amount of thiosulfate into chloride solutions could lower the pitting potential and increase the dissolution rate of alloy 800. At pitting potential, the dissolution of metal ions from pitting is not remarkable in chloride-only solution, whereas dissolution of metal ions is significant in thiosulfate and chloride solution.

Acknowledgments This work was supported by Natural Sciences and Engineering Research Council of Canada Discovery Grant and National Natural Science Foundation of China (No. 51371124). The authors also gratefully acknowledge R.L. Tapping, P. Angell, Yucheng Lu and Stan J. Klimas of AECL for supporting this work.

\section{References}

[1] L. Fan, Z. Y. Liu, W. M. Guo, J. Hou, C.W. Du, X.G. Li, Acta Metall. Sin. (Engl. Lett.) 28, 866 (2015)

[2] A. Parsapour, S. Khorasani, M. Fathi, Acta Metall. Sin. (Engl. Lett.) 26, 409 (2013)

[3] Y. Zhang, B. Chen, Z. Guo, Acta Metall. Sin. (Engl. Lett.) 27, 331 (2014)

[4] D.H. Xia, L.X. Yang, Acta Phys-Chim Sin. 30, 1465 (2014)

[5] D.H. Xia, S.Z. Song, W.Q. Gong, Y.X. Jiang, Z.M. Gao, J.H. Wang, J. Food Eng. 113, 11 (2012)
[6] D.H. Xia, S.Z. Song, J.H. Wang, H.C. Bi, Z.W. Han, Trans. Tianjin Univ. 18, 15 (2012)

[7] C. Thee, L. Hao, J.H. Dong, X. Mu, W. Ke, Acta Metall. Sin. (Engl. Lett.) 28, 261 (2015)

[8] J. Li, H.J. Zheng, Y. Zheng, W.K. Kong, K. Wang, Acta Metall. Sin. (Engl. Lett.) 28, 449 (2015)

[9] N. Cui, H.Y. Ma, J.L. Luo, S. Chiovelli, Electrochem. Commun. 3, $716(2001)$

[10] R.K. Zhu, J.L. Luo, Electrochem. Commun. 12, 1752 (2010)

[11] R.K. Zhu, B.T. Lu, J.L. Luo, Y.C. Lu, Appl. Surf. Sci. 270, 755 (2013)

[12] D.H. Xia, H.Q. Fan, L.X. Yang, Y. Behnamian, J.L. Luo, Y.C. Lu, S. Klimas, J. Electrochem. Soc. 162, C482 (2015)

[13] D.H. Xia, R.K. Zhu, Y. Behnamian, J.L. Luo, C.J. Lin, S. Klimas, J. Electroanal. Chem. 744, 77 (2015)

[14] R. Zhao, Z. Zhang, J.B. Shi, L. Tao, S.Z. Song, J. Cent, South Univ. 17, 13 (2010)

[15] L. Li, C. Wang, S.H. Chen, X.G. Yang, B.Y. Yuan, H.L. Jia, Electrochim. Acta 53, 3109 (2008)

[16] B. Yuan, C. Wang, L. Li, S. Chen, Electrochem. Commun. 11, 1373 (2009)

[17] D.H. Xia, J.L. Luo, Trans. Tianjin Univ. 21, 234 (2015)

[18] L. Li, C. Wang, B. Yuan, S. Chen, Electrochem. Commun. 10, 103 (2008)

[19] D.H. Xia, S.Z. Song, R.K. Zhu, Y. Behnamian, C. Shen, J.H. Wang, J.L. Luo, Y.C. Lu, S. Klimas, Electrochim. Acta 111, 510 (2013)

[20] B. Yuan, W. Li, C. Wang, L. Li, Sensors Actuators B 176, 509 (2013)

[21] D.H. Xia, Y. Behnamian, H.N. Feng, H.Q. Fan, L.X. Yang, C. Shen, J.L. Luo, Y.C. Lu, S. Klimas, Corros. Sci. 87, 265 (2014)

[22] R.C. Newman, H.S. Isaacs, B. Alman, Corrosion 38, 261 (1982)

[23] D.H. Xia, R.K. Zhu, Y. Behnamian, C. Shen, J.L. Luo, Y.C. Lu, S. Klimas, J. Electrochem. Soc. 161, C201 (2014) 